\section{Original article}

\title{
Home screening for chlamydial genital infection: is it acceptable to young men and women?
}

\author{
Judith Stephenson, Caroline Carder, Andrew Copas, Angela Robinson, Geoffrey Ridgway, \\ Andrew Haines
}

Objectives: To determine the acceptability, to young men and women, of home screening for chlamydial infection.

Methods: We wrote to a random sample of 208 women aged 18-25 years and 225 men aged 18-35 years from three general practices, inviting them to undergo home screening for chlamydial infection. They were asked to return, by normal post, a urine specimen (for men and half of the women) or a vulval swab (other half of the women) for ligase chain reaction (LCR) testing for chlamydial infection. They were also asked to return a short questionnaire about risk status and the acceptability of this approach.

Results: The participation rate among the available sample was $39 \%$ for women and $46 \%$ for men $(p=0.3)$. However, among women, the rate was slightly higher $(p=0.05)$ for urine samples $(47 \%)$ than for vulval swabs (32\%). Six per cent of women and $9 \%$ of men declined to take part, while $42 \%$ of women and $33 \%$ of men failed to respond. Two men objected to receiving the package at home. We received few other comments, positive and negative in about equal measure.

Conclusion: Home screening for chlamydial infection is a potentially efficient method of reaching young people who may have little contact with health services. Men were at least as likely as women to respond to this screening approach. Home screening might form a useful component of a future chlamydial screening programme in the United Kingdom.

(Sex Transm Inf 2000;76:25-27)

Keywords: chlamydial infection; screening; non-invasive sampling

\section{Introduction}

Screening for chlamydial genital infection is generating much interest, especially since the advent of self administered and non-invasive sampling methods. ${ }^{12}$ The most important research question identified in a recent UK government report is how to maximise the cost effectiveness of screening in non-genitourinary clinic settings, including which test and specimen to use for screening men and women. ${ }^{3} \mathrm{~A}$ number of self administered methods, including vaginal, vulval, and urine sampling have been evaluated favourably in comparison with more invasive methods that cannot be self taken, such as endocervical samples. ${ }^{1}{ }^{4}$ Vulval swabs and urine specimens are both noninvasive methods with high sensitivity to ligase chain reaction (LCR) testing for chlamydia. ${ }^{5}$ Vulval swabs have the advantage over urine specimens of requiring less processing in the laboratory and therefore being cheaper, but the relative acceptability of these two methods to women is not clear. We therefore investigated the acceptability and feasibility of home screening for chlamydial infection using urine samples for men, and urine samples or vulval swabs for women.

\section{Methods}

The study was approved by local ethics committees. It was conducted with three general practices that were linked to research networks. We included two urban practices in North London and one in Avon, as we wanted to see whether the participation rate would be higher outside London. In each practice we wrote to a random sample of women aged 18-25 years and men aged $18-35$ years (totalling 208 women and 225 men), registered with the practices, asking them to take part in a research study about home screening for chlamydia. (The age range was older for men to reflect differences in the age distribution of chlamydia reported from genitourinary medicine clinics.) The letter made clear that this was a research project and was signed by the general practitioner and one of the researchers (JS) on joint headed paper. It included a leaflet with information about chlamydial infection, questions about risk status, including whether they had ever had sexual intercourse, questions about the acceptability of this approach, and a "home testing kit" with instructions and packaging for return of samples to the study laboratory via normal (first class) post. The packaging conformed to Royal Mail packaging regulations which stipulates three layers of packaging: a primary, labelled, watertight, leak proof receptacle containing the sample and wrapped in enough absorbent material to absorb all fluid in case of breakage; a second watertight, leak proof receptacle to enclose the primary one, and an outer package to protect against external damage during transit. The study sample size was chosen to enable the overall participation rate to be estimated within $5 \%$ of the true value, where the true value was assumed to lie in the range $30 \%-60 \%$.

The leaflet explained that people who had never had sexual intercourse were not at risk of chlamydial infection; they were asked to complete the questions, but not to send a sam-
Accepted for publication 5 November 1999 
Table 1 Results for the three practices combined

\begin{tabular}{|c|c|c|c|}
\hline & $\begin{array}{l}\text { Women (swab) } \\
\text { No }(\%)\end{array}$ & $\begin{array}{l}\text { Women (urine) } \\
\text { No }(\%)\end{array}$ & $\begin{array}{l}\text { Men } \\
\text { No (\%) }\end{array}$ \\
\hline Total sample & $105(100)$ & $103(100)$ & $225(100)$ \\
\hline \multicolumn{4}{|l|}{ Outcome } \\
\hline participated & $27(26)$ & $38(37)$ & $80(36)$ \\
\hline declined & $7(7)$ & $6(6)$ & $21(9)$ \\
\hline no response & $51(49)$ & $37(36)$ & $74(33)$ \\
\hline "ghosts" & $20(19)$ & $22(21)$ & $50(22)$ \\
\hline Available sample & $85(100)$ & $81(100)$ & $175(100)$ \\
\hline Participated & $27(32)$ & $38(47)$ & $80(46)$ \\
\hline Among participants & All women & & Men \\
\hline Ever had sexual intercourse & $48(76)$ & & $69(86)$ \\
\hline Among those reporting intercourse & $48(100)$ & & $69(100)$ \\
\hline \multicolumn{4}{|l|}{ No of partners in past year } \\
\hline-0 & $1(2)$ & & $2(3)$ \\
\hline-1 & $30(63)$ & & $50(73)$ \\
\hline-2 & $9(19)$ & & $5(7)$ \\
\hline $3+$ & $8(17)$ & & $11(16)$ \\
\hline Previous STD & $9(19)$ & & $6(9)$ \\
\hline Positive chlamydia test result & $4(8)$ & & $2(3)$ \\
\hline
\end{tabular}

"Ghosts" are people no longer living at the address held by the practice.

*Among those who had ever had sexual intercourse.

†Chlamydia, gonorrhoea, genital herpes, genital warts, and PID (pelvic inflammatory disease) or NSU (non-specific urethritis) for women and men respectively.

ple. All the men, and a random $50 \%$ sample of the women, were sent a urine testing kit: the other $50 \%$ of the women were sent a vulval swab (cotton wool tip with a plastic stem) and tube of buffer solution (provided by Abott, UK). Each package contained an illustrated set of instructions on how to take the sample and how to package it for mailing (copies available from authors). To take the vulval swab, women were asked to "remove the swab from the packet labelled swab. With one hand, open the folds of skin around the vagina. Take the swab in your other hand and wipe the cotton wool end around the entrance to the vagina."

In London, a repeat package was sent 3 weeks later to non-responders by recorded delivery to distinguish genuine non-responders from "ghosts" (that is, people no longer living at the address held by the practice). In Avon, both first and repeat packages were sent by recorded delivery. All specimens were tested for chlamydial infection using the LCR. ${ }^{1}$ Ninety one per cent of specimens were received at the laboratory within 4 days of being taken; the maximum time was 7 days. The data were analysed approximately 5 months after the first invitation to screening was sent out. No further responses were received after this time. For statistical analysis, we used the $\chi^{2}$ test to assess whether the participation rates were significantly different between population groups.

\section{Results}

The overall participation rate was 31\% (95\% CI $25-38 \%)$ in women and $36 \%(29-42 \%)$ in men (table 1). The participation rate among the available sample (excluding "ghosts") was $39 \%(31-47 \%)$ for women and $46 \%(38-53 \%)$ for men $(p=0.3)$. However, among women, the rate was somewhat higher $(\mathrm{p}=0.05)$ for urine samples $(47 \%$, 95\% CI 36-58\%) than for vulval swabs $(32 \%, 22-43 \%)$. Nearly two thirds $(62 \%)$ of responses were received after the first screening invitation, with the remainder $(38 \%)$ arriving after the sending of a second invitation. Participation rate was unrelated to age among either men or women. The median age of male participants was 27 compared with 25 in non-participants, and for women the median age of both participants and non-participants was 22 years. Participation rate was higher in the Avon practice, compared with the two London practices which had similar participation rates. The rates in men were $58 \%(32 / 55)$ in Avon compared with 40\% (48/120) for the two London practices combined $(\mathrm{p}=0.04)$ and $50 \%(30 / 60)$ versus 33\% (35/106) in women $(\mathrm{p}=0.05)$ respectively. The six people with a positive test result were contacted and advised to have treatment and contact tracing.

In response to specific questions about the acceptability of this approach, there were no negative responses to being asked sensitive questions. Three women were unsure whether they had taken the vulval swab correctly. Another woman commented that taking the swab was "quite personal." One man reported getting urine on his hands, but didn't mind doing this at home, and one women wrote that it was difficult to collect the first part of the urine stream. Two men objected to receiving the package at home, particularly by recorded delivery, and suggested that people should have the opportunity to decline beforehand. Four people volunteered positive comments about participating.

\section{Discussion}

Home screening for chlamydial infection is a potentially efficient method of reaching young people who have little contact with health services. Around half the available sample responded (participated or declined) swiftly to this approach, and men were as likely as women to take part. Unlike previous studies of home sampling, ${ }^{3}$ the only contact with our target group was by post - there was no meeting with healthcare workers beforehand. Among women, the response rate to vulval swabs was slightly lower than to urine samples, but since we did not offer women the choice between urine or vulval sample we cannot conclude that either method is clearly preferable.

We cannot tell whether non-response from the other half of the target population indicated disapproval of this approach or lack of interest in screening. Although the study was not designed to estimate the prevalence of chlamydial infection, the frequency of current infection and history of a previous sexually transmitted infections among responders may suggest that those at high risk of chlamydial infection were more likely to respond. An alternative approach might be to send a letter of introduction, giving people the opportunity to decline to receive a screening kit.

The Department of Health is piloting the feasibility of opportunistic screening of women attending general practice. Home screening could offer substantial savings, to individuals and the health service, because direct contact with healthcare professionals is limited to the few who screen positive. Home screening could form a useful component of a community based chlamydial screening programme in which non-responders could be offered opportunistic screening at the general practice. 
We thank all the patients and general practice staff from Eagle House Surgery, Enfield, the Oakwood Medical Centre, North London and Surgery 18 in Bath who took part in the study.

Contributors: JS devised the idea for the study, designed the protocol, and wrote the first draft of the manuscript; CC and GR were responsible for receiving specimens and for LCR testing for chlamydial infection; AR advised on the management of people with chlamydial infection; $\mathrm{AH}$ advised on recruitment of general practices; and AC conducted the statistical analysis; al authors contributed to various drafts of the manuscript.

1 Grun L, Tassano-Smith J, Carder C, et al. Comparison of two methods of screening for genital chlamydial infection in women attending in women attending in gen
2 Ostergaard L, Andersen B, Olesen F, et al. Efficacy of home sampling or screening of Chlamydia trachomatis: randomised study. BMF 1998;317:26-27.

3 Department of Health. Chlamydia trachomatis. Summary and conclusions of CMO's Expert Advisory Group. London: DoH, 1998.

4 Ostergaard L, Moller JK, Andersen B, et al. Diagnosis of urogenital Chlamydia trachomatis infection in women based on mailed samples obtained at home: multipractice comparative study. BMF 1996;313:1186-9.

5 Stary A, Najim B, Lee HH. Vulval swabs as alternative specimens for ligase chain reaction detection of genital chlamydial infection in women. $\mathcal{F}$ Clin Microbiol 1997;35: 836-8. 